\title{
Exposition « Sur les chemins de l'Atlantide : Leo Frobenius (1873-1938) et l'art rupestre africain »
}

\section{Hélène Ivanoff}

\section{(2) OpenEdition}

\section{Journals}

Édition électronique

URL : http://journals.openedition.org/ifha/7994

DOI : $10.4000 /$ ifha.7994

ISSN : 2198-8943

\section{Éditeur}

IFRA - Institut franco-allemand (sciences historiques et sociales)

\section{Édition imprimée}

Date de publication : 31 décembre 2014

ISSN : 2190-0078

\section{Référence électronique}

Hélène Ivanoff, «Exposition « Sur les chemins de l'Atlantide : Leo Frobenius (1873-1938) et l'art rupestre africain » », Revue de l'IFHA [En ligne], 6 | 2014, mis en ligne le 31 décembre 2014, consulté le 19 avril 2019. URL : http://journals.openedition.org/ifha/7994 ; DOI : 10.4000/ifha.7994

Ce document a été généré automatiquement le 19 avril 2019

(C)IFHA 


\section{Exposition « Sur les chemins de} l'Atlantide : Leo Frobenius (1873-1938) et l'art rupestre africain »

Hélène Ivanoff

ill. 1 : Entrée de l'exposition

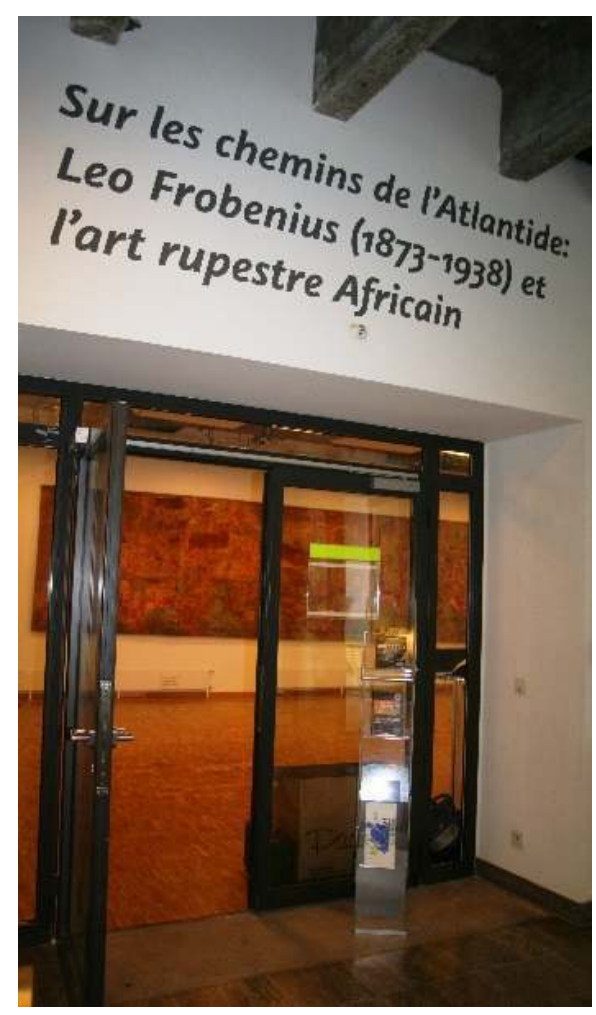




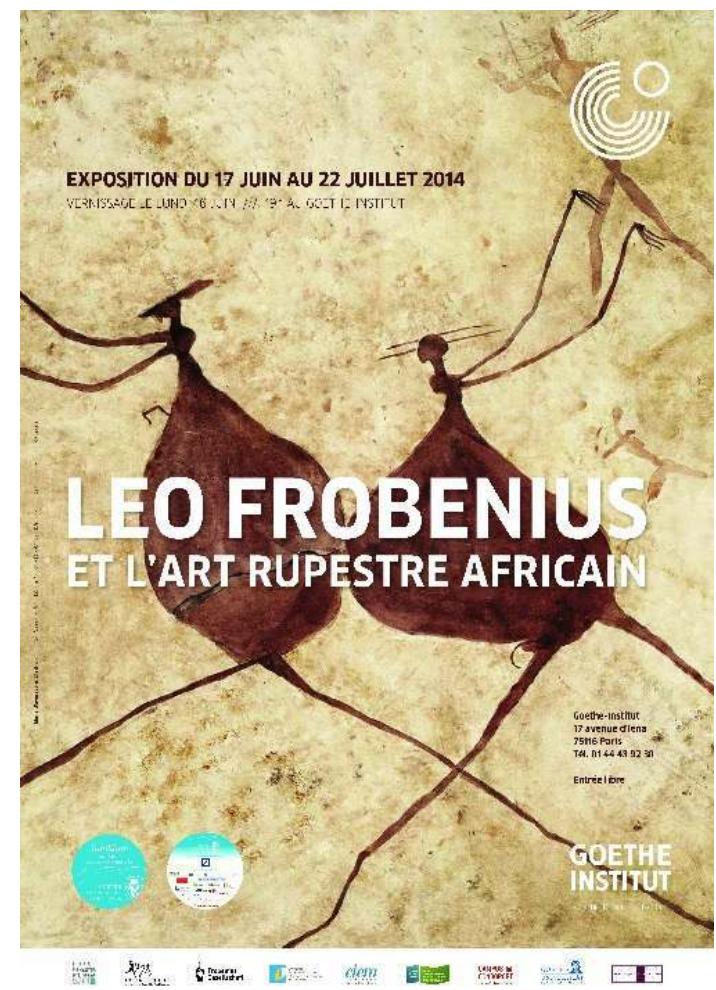

Présentée à l'Institut Goethe de Paris du 16 juin au 22 juillet 2014, l'exposition "Sur les chemins de l'Atlantide: Leo Frobenius (1873-1938) et l'art rupestre africain» est récemment venue apporter de nouveaux éclairages sur le parcours de cet étonnant explorateur que fut Leo Frobenius et les collections qu'il rassembla au cours de ses expéditions africaines dans la première moitié du vingtième siècle (ill. 1). Cet ethnologue allemand fut fasciné dès son plus jeune âge par les récits de son grand-père, Heinrich Bodinus, lui contant les voyages d'exploration à l'intérieur de l'Afrique de Carl Peters, Heinrich Barth, Gustav Nachtigal, Hermann von Wissmann ou Paul Pogge. Directeur du jardin zoologique de Berlin, ce dernier lui fit aussi connaître l'Afrique au travers des spectacles exotiques, organisés dans la métropole coloniale. Employé par les musées d'ethnographie de Brême, Bâle et Leipzig, il élabora des théories sur l'art de peuples dits " naturels ", Naturvölker, à partir de l'analyse des collections ethnographiques conservées dans l'Empire allemand. En marge des milieux académiques, il fonda en 1898 ses archives africaines, Afrika Archiv, puis un institut de morphologie culturelle, Institut für Kulturmorphologie, à Munich en 1920, transféré ensuite à Francfort en 1925, où il devint finalement professeur puis directeur du musée ethnographique de la ville en 1934.

Organisée à l'occasion du centenaire de la fondation de l'université Goethe de Francfort en coopération avec la société des amis de l'Institut Frobenius, l'Institut français d'histoire en Allemagne (IFHA), le Centre Georg Simmel (CNRS-UMR 8131) de l'EHESS, le Campus Condorcet et le Centre interdisciplinaire de recherches et d'études sur l'Allemagne (CIERA), l'exposition parisienne fut pour l'Institut Frobenius de Francfort l'opportunité de présenter ses collections universitaires hors les murs (ill. 2).

3 Les trois commissaires d'exposition - Jean-Louis Georget, Hélène Ivanoff, Richard Kuba ont choisi de montrer leur variété et leur richesse, de les replacer dans le contexte 
culturel de cette époque coloniale et de mettre en exergue tant leurs valeurs scientifiques qu'artistiques. Simultanément à cette exposition, des conférences ont été proposées à l'EHESS et à l'Institut Goethe, grâce au soutien de l'Université franco-allemande ${ }^{1}$. Elles ont quant à elles été centrées sur l'apport de Leo Frobenius dans la constitution d'un savoir africaniste et ont été consacrées, dans une approche transnationale plus large, à la constitution d'un savoir africaniste et à l'histoire de l'institutionnalisation de l'ethnologie en France, aux États-Unis et dans les pays de langue allemande. Alors que les relevés d'Henri Lothe établis entre 1956 et 1970 dans les monts du Tassili n'Ajjer du Sahara central étaient parallèlement exposés au Musée de Louviers et tandis que la grotte Chauvet entrait au patrimoine de l'humanité, il était temps de revenir sur le parcours de cet africaniste allemand qui rassembla, dès avant 1914 et principalement dans les années 1930, les traces d'un art préhistorique africain.

\section{ill. 3 : Salle 1 de l'exposition}

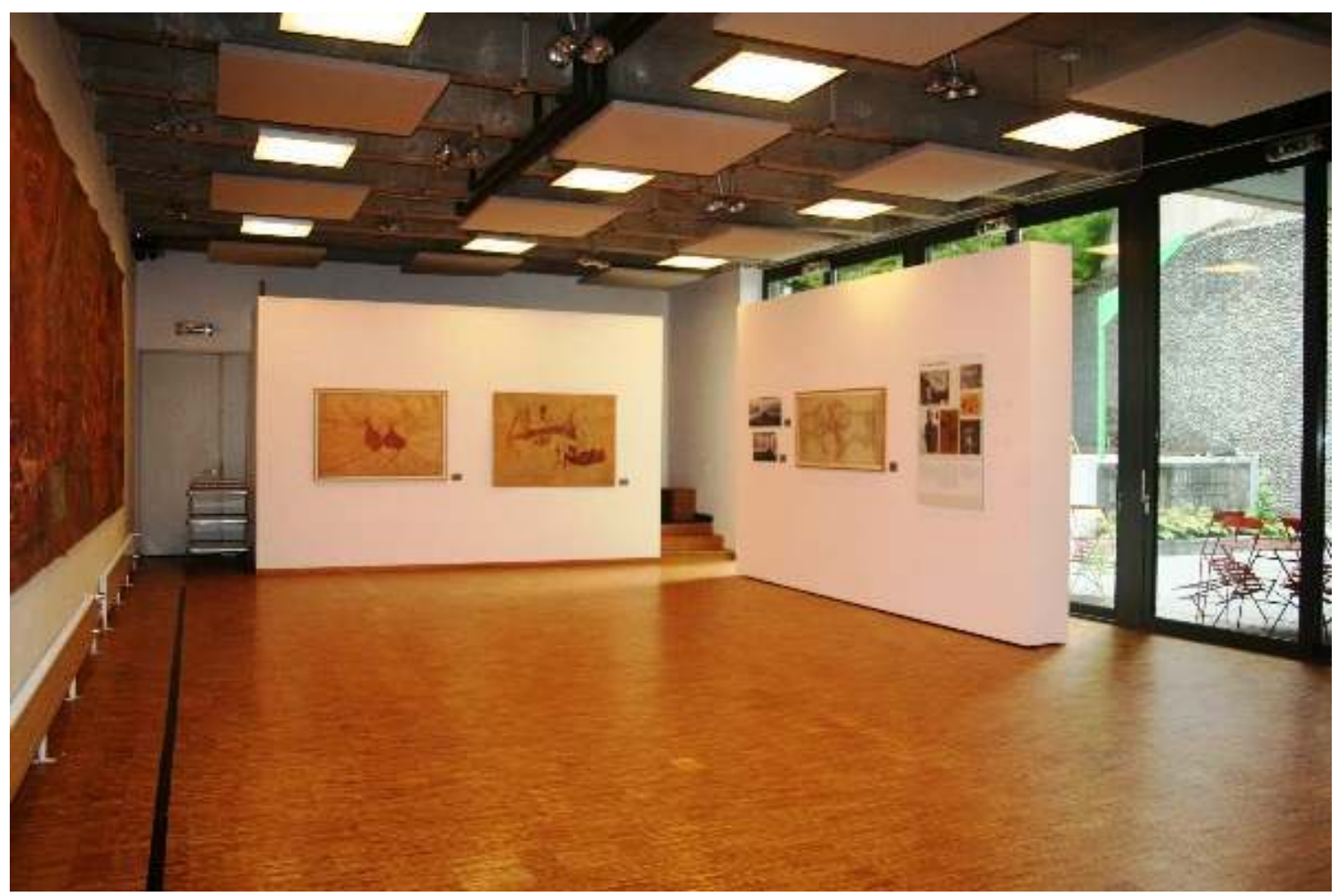


ill. 4 : Salle 2 de l'exposition

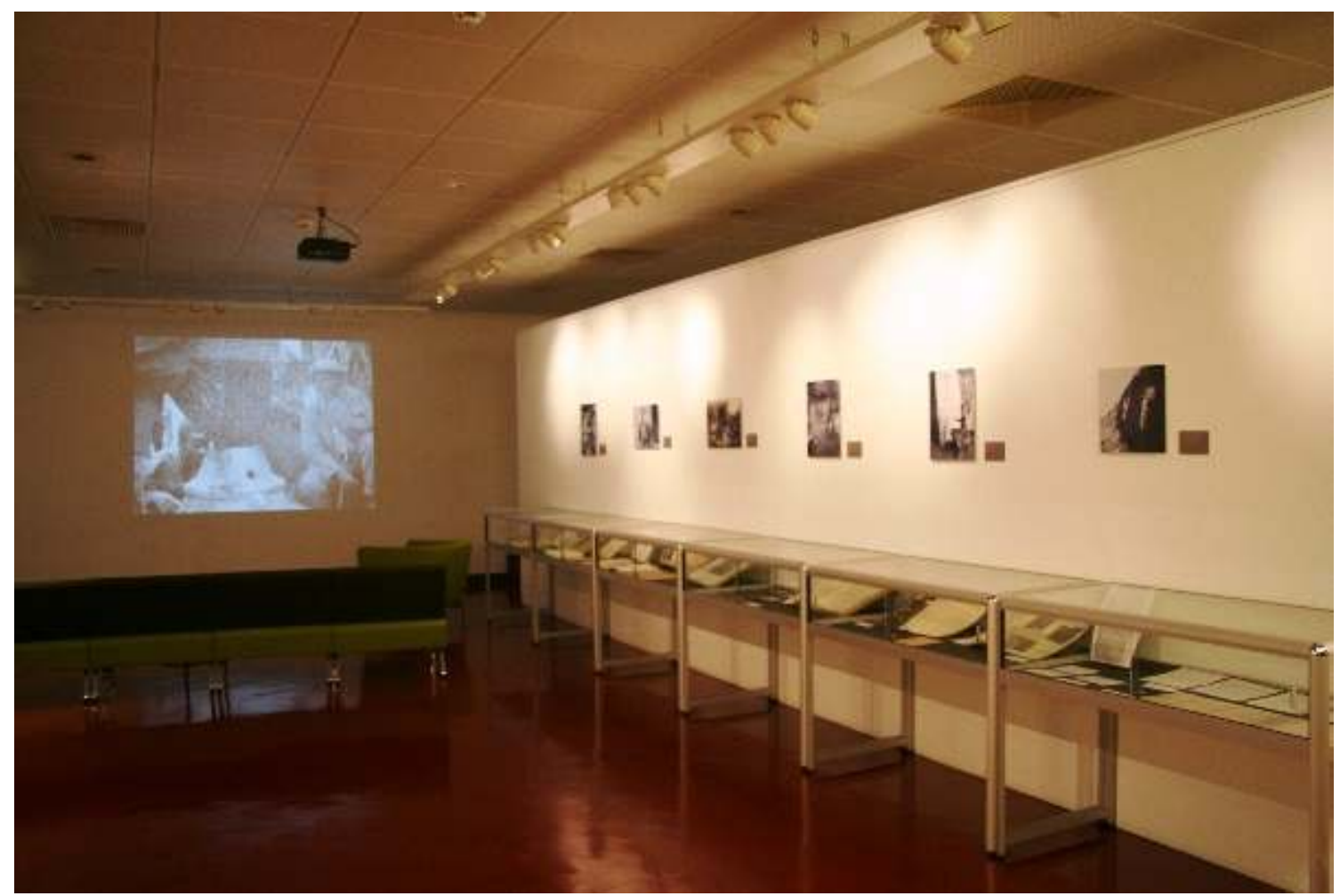

ill. 5 : Corridor de l'exposition

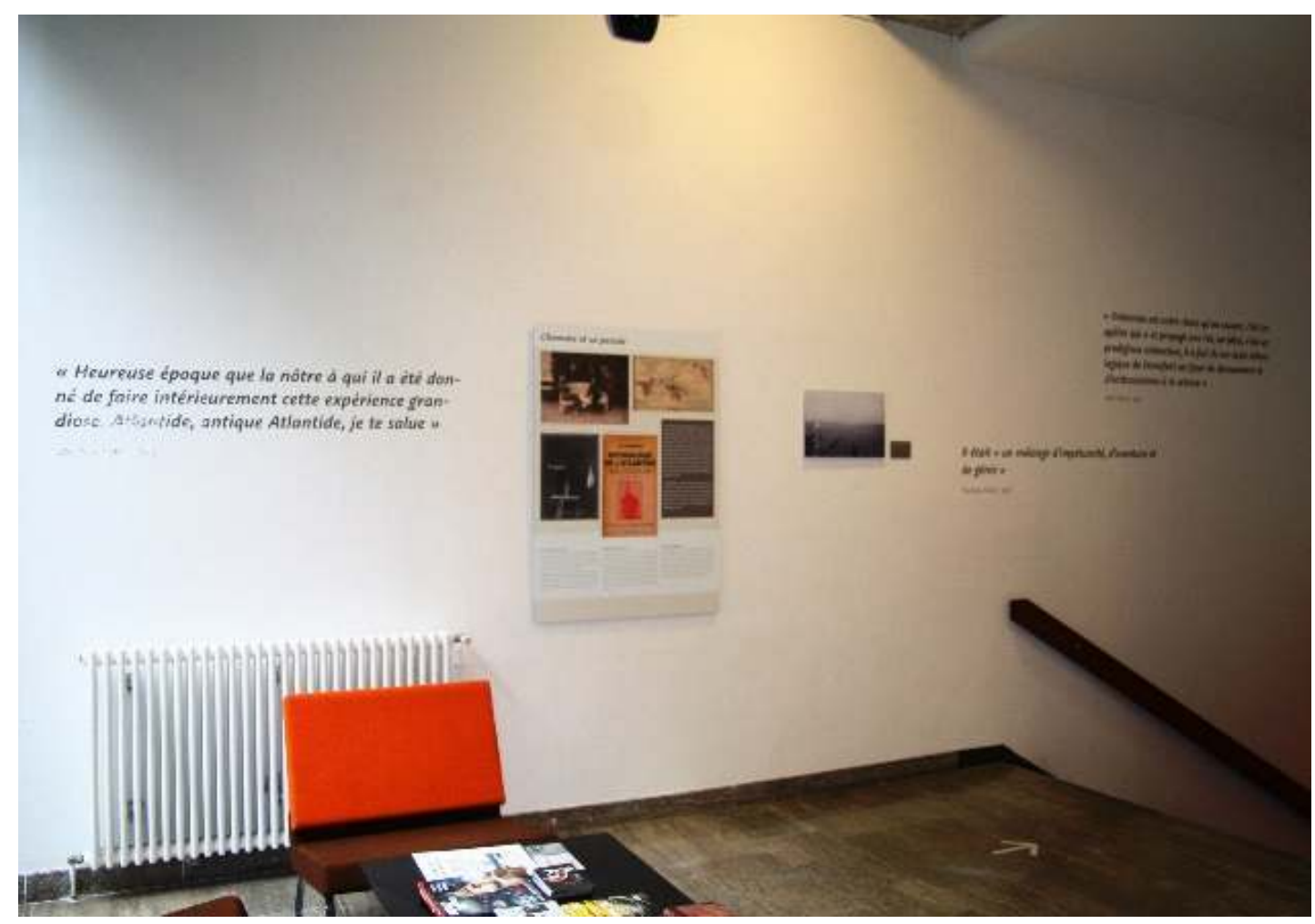




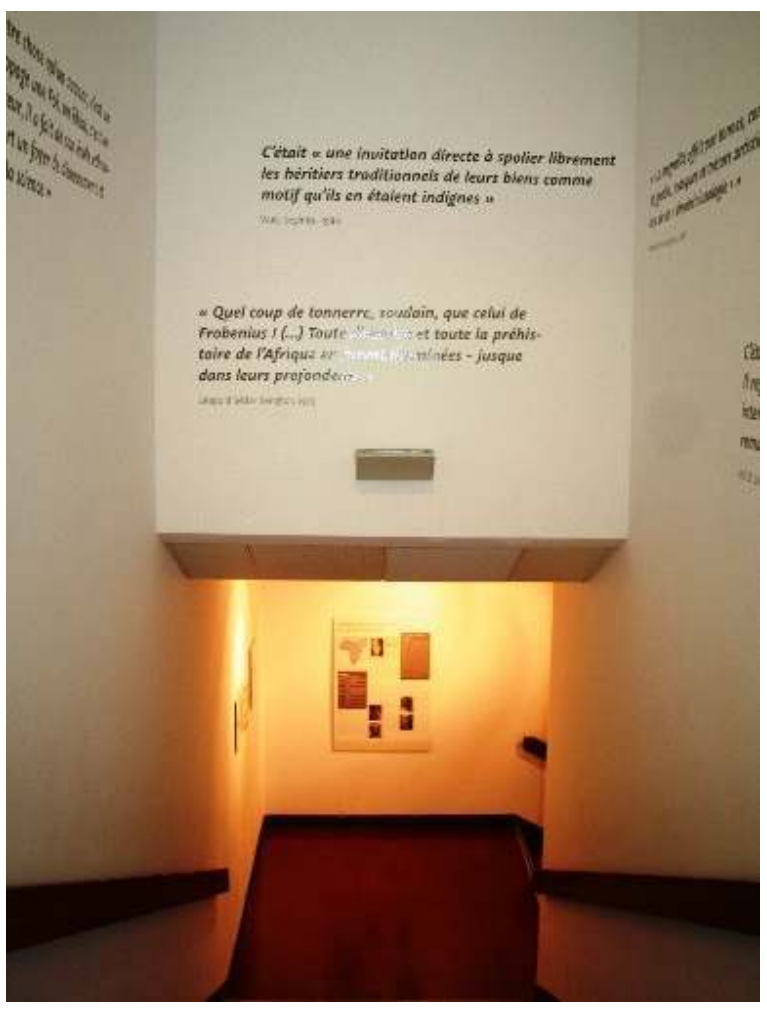

4 L'exposition de l'Institut Goethe proposait aux visiteurs un parcours centré autour de deux salles principales. La première réunissait des aquarelles sur papier et huiles sur toile, photographies, dessins au crayon et copies d'art rupestre africain déjà exposées en Europe et aux États-Unis dans les années 1930 (ill.3). La seconde rendait compte de la vaste documentation ethnographique réunie par Leo Frobenius lors de ses expéditions dans l'Afrique coloniale et montrait comment ce savoir africaniste avait été constitué et reçu par ses contemporains (ill.4). Entre ces deux salles, le visiteur était invité à s'interroger sur la pensée de Leo Frobenius (ill.5) et la portée de son œuvre auprès des scientifiques, écrivains et intellectuels, notamment africains (ill.6). Refusant l'hagiographie ou l'héroïsation de cet africaniste allemand, l'exposition insistait au contraire sur l'ambivalence de ce personnage. Critiqué pour ses méthodes scientifiques et ses collectes massives en Afrique, il fut cependant à l'origine d'un nouveau regard sur l'histoire et la culture africaine à l'époque coloniale. Après avoir souligné le statut ambigu de ces copies d'art rupestre, dû en outre à l'histoire de leurs expositions, nous reviendrons sur les expéditions de Leo Frobenius et les aspects controversés et polémiques de sa pensée présentés lors de cette exposition.

\section{Images d'un art préhistorique africain}


ill. 7 : Maria Weyersberg et Elisabeth Mannsfeld, Style classique du Sud, peint en plusieurs couches, 1929, Aquarelle sur papier, 2,10 x 10,73 m, Copie d'art rupestre faite à Ha Baroana, Lesotho.

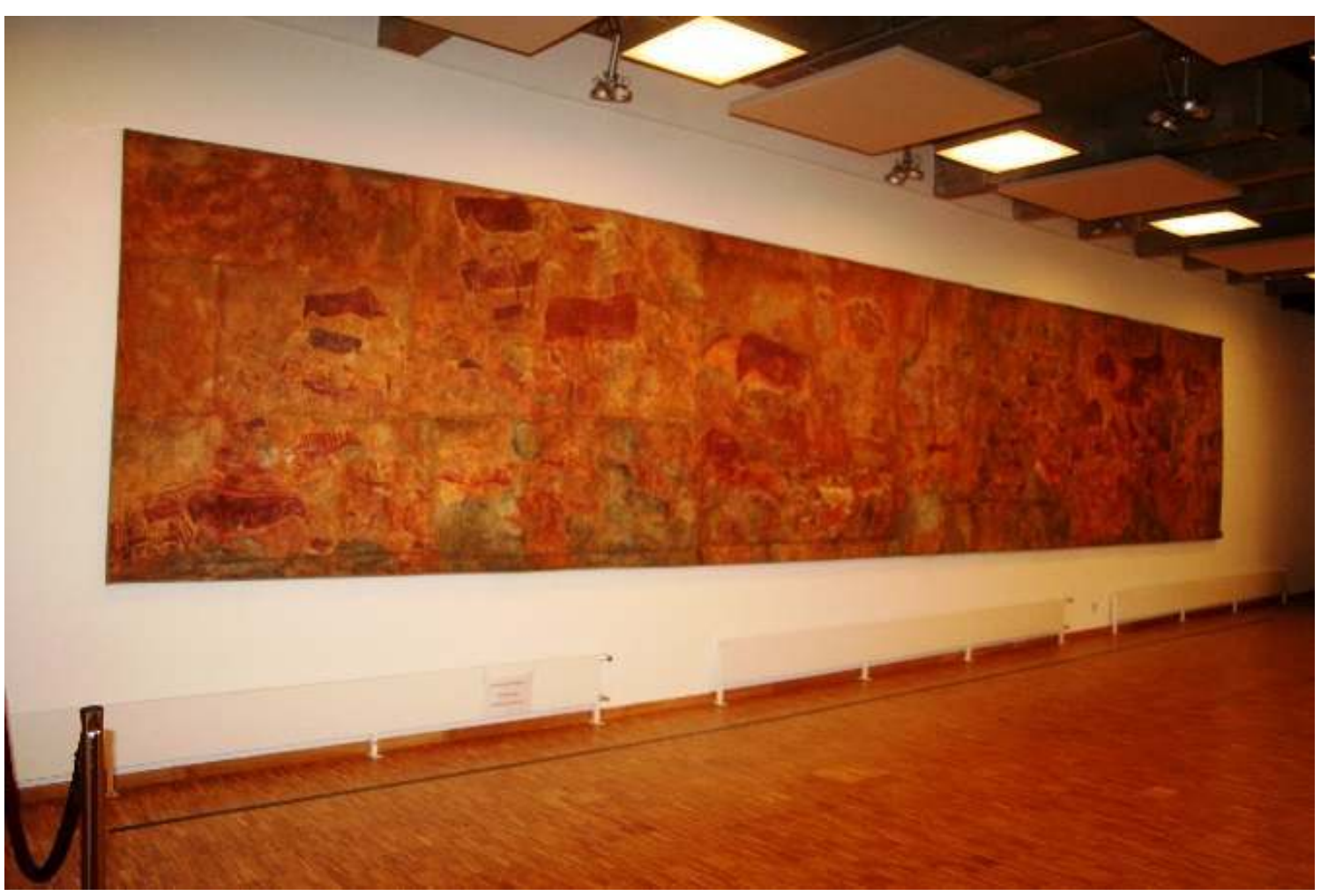

ill. 8 : Détail

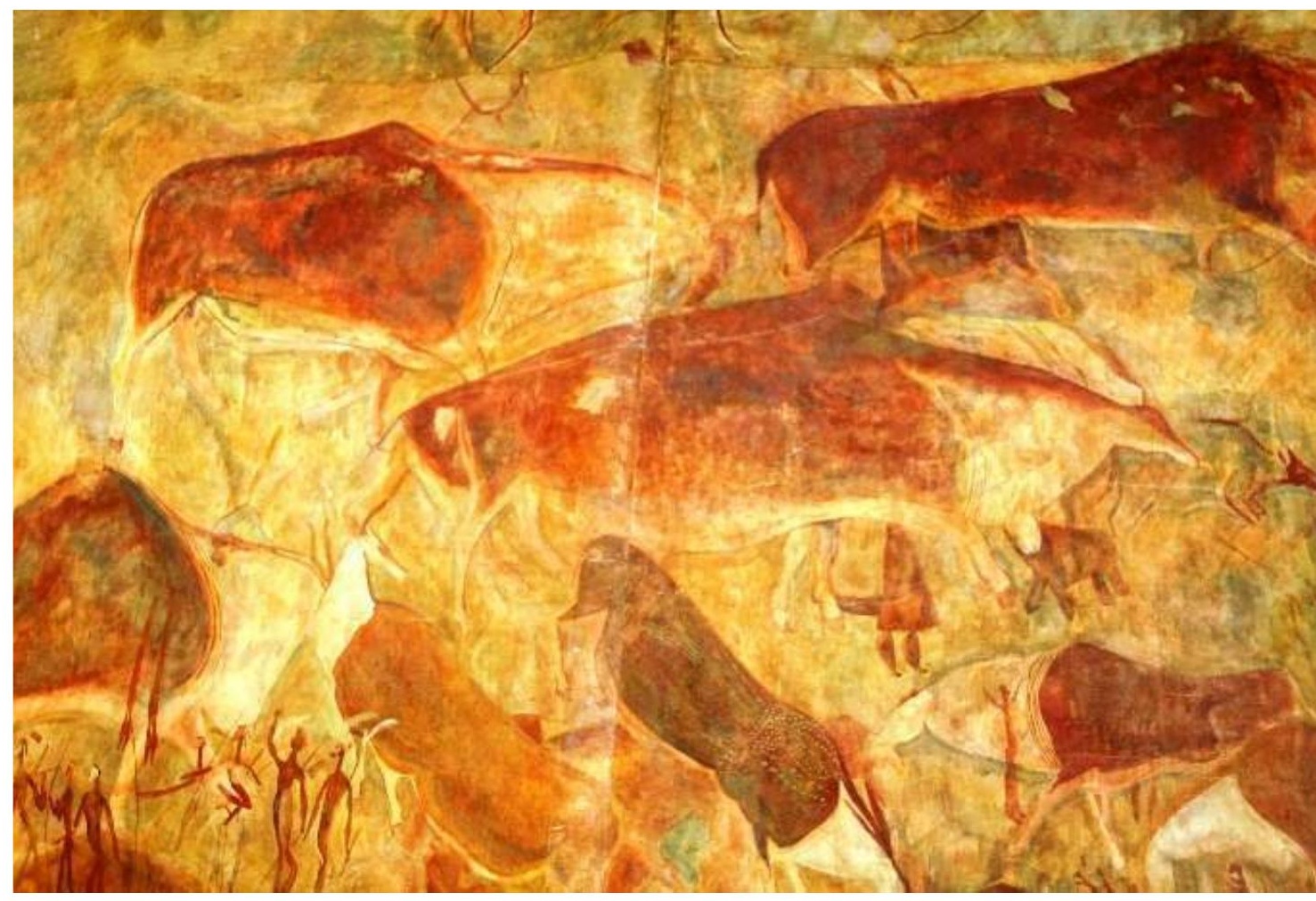


Les images rupestres de l'Institut Frobenius donnent à voir les peintures et gravures observées sur les rochers d'Afrique du Sud et du Sahara occidental dans la première moitié du XXe siècle. Elles furent établies par Leo Frobenius et les artistes qui l'accompagnèrent lors de ses voyages. Parmi elles, une grande aquarelle sur papier, d'environ deux mètres sur dix, saisit d'emblée le visiteur de l'exposition (ill. 7). Elle fut réalisée par Maria Weyersberg et Elisabeth Mannsfeld en 1929, d'après les peintures couvrant les abris sous roche de Ha Baroana dans les montagnes du Drakensberg, sur l'actuel territoire du Lesotho. Elle représente des élans du Cap, grandes antilopes des savanes africaines, des boucs, et des processions d'hommes tenant des trompes et des emblèmes, ainsi que quelques scènes de chasse, dans des tons ocre, vert et brun. D'aspect figuratif, ces représentations surprennent cependant d'une part par la taille imposante des figures animales, aux corps surdimensionnés par rapport aux têtes, et d'autre part par la simplification des figures humaines, simples silhouettes, marchant et courant arcs en main, se fondant totalement dans ce milieu naturel (ill. 8). D'après Leo Frobenius, ces peintures rupestres étaient représentatives d'un style classique du Sud en plusieurs couches, typique du Basutoland, les plus anciennes datant de plusieurs millénaires. Les attribuant aux San, les recherches actuelles tendent à prouver qu'en Afrique du Sud les peintures rupestres seraient en fait vieilles d'au moins 4000 ans.

ill. 9 : Sur le plan incliné : Ruth Assisa Cuno, Composition animale, 1932, Huile sur toile, 2,75 x 2,05 m, Copie d'art rupestre du Fezzan, Lybie.

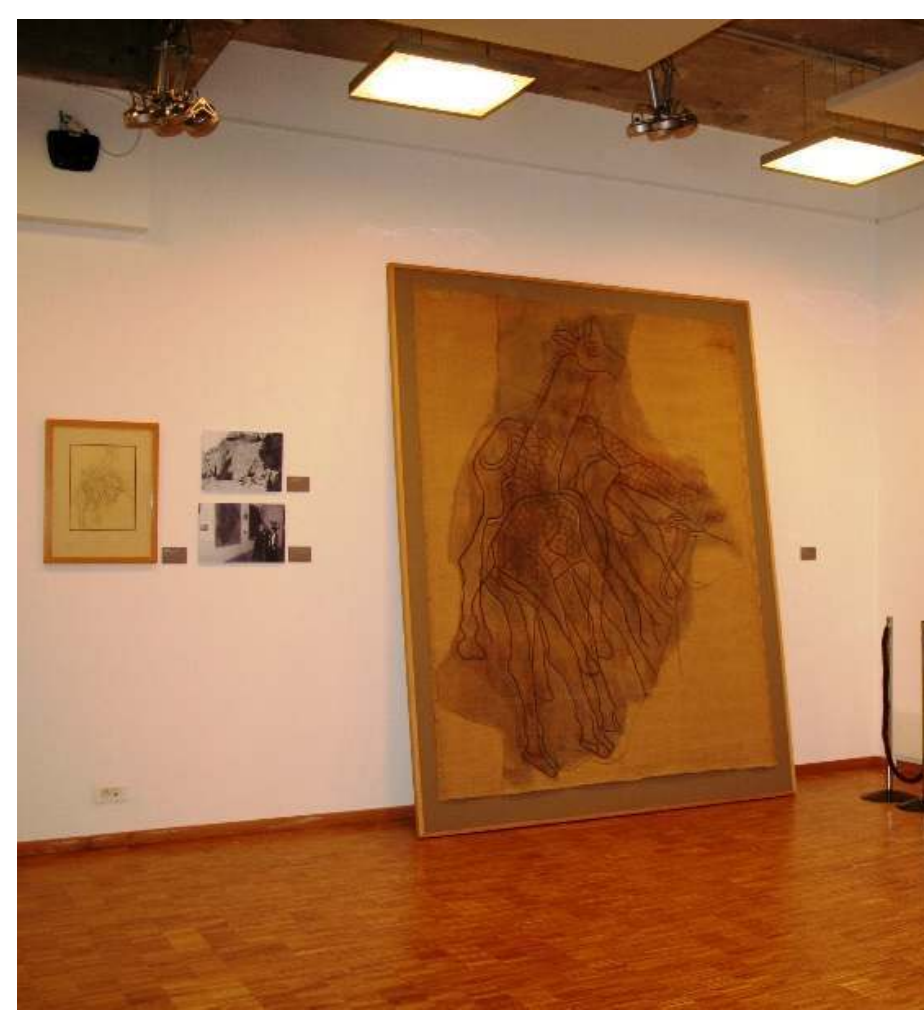




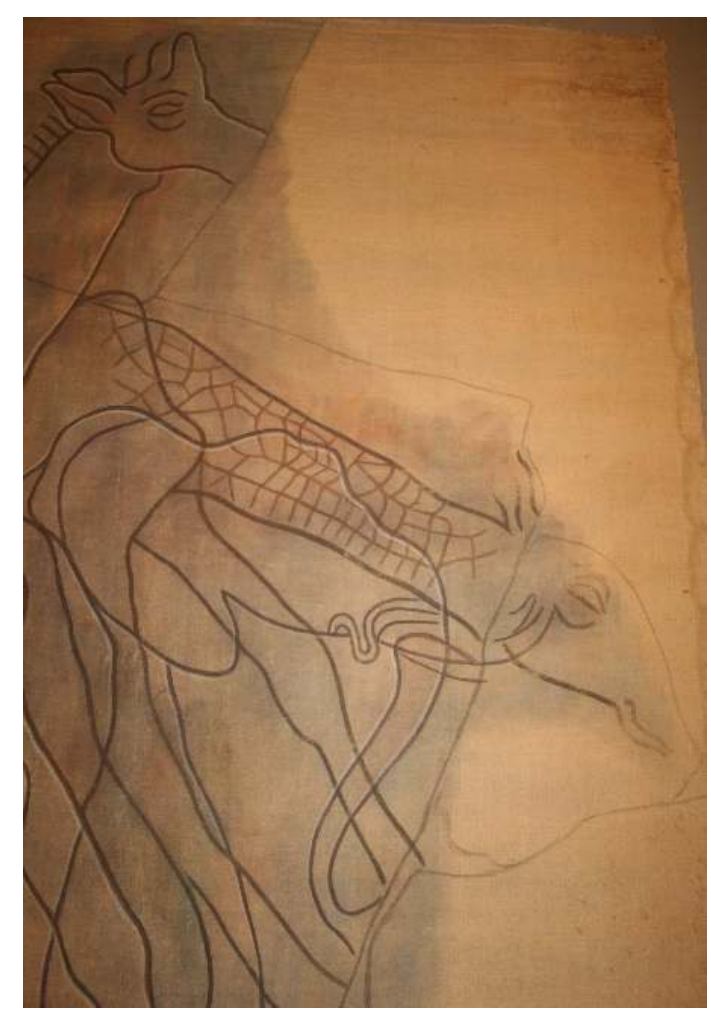

Dans cette exposition, un autre grand format frappait aussi par son aspect monumental : une huile sur toile, de trois mètres sur deux, de Ruth Assisa Cuno (ill. 9). Dans cette composition animale, deux girafes et un éléphant sont enchevêtrés, à l'image de la gravure rupestre originale, située sur un rocher en plein air qui servit de modèle. En attestent la photographie et le dessin, placés dans la salle d'exposition aux côtés de cette toile, dressée sur un plan incliné. Copiées dans le Fezzan, dans le wadi in Habeter III (Mathendous) en 1932, ces gravures rupestres du Sud-Ouest de la Lybie actuelle dateraient de 10 à 12000 ans avant notre ère, certains spécialistes militant eux pour un âge plus récent, entre 6000 à 7000 avant J.-C. À la surprise que provoque cette superposition de figures animales, dont le spectateur doit suivre le tracé noir de l'artiste pour reconstituer les contours, s'ajoute le fait que certaines parties du dessin sont manquantes, tel le museau de la girafe, disparu dès 1932 avec le rocher qui portait cette gravure, en raison de l'érosion (ill.10). Ce relevé avait été exposé en 1933 au musée d'ethnographie du Trocadéro. Sur la photographie de l'exposition, Georges-Henri Rivière, accompagné d'un couple de visiteurs, tentait lui aussi de déchiffrer cette image qui ne se laisse que difficilement saisir au premier coup d'œil. ${ }^{2}$ 
ill. 11 : À droite Agnès S. Schulz, Tombeau du roi, style classique, 1928-1930, Aquarelle sur papier, $112 \times 146 \mathrm{~cm}$, Copie d'art rupestre du Zimbabwe. À gauche Maria Weyersberg, Mantes religieuses, 1928-1930, Aquarelle sur papier, 92 x $135 \mathrm{~cm}$, Copie d'art rupestre de Namibie.

7 Les autres aquarelles en couleurs (ill. 11), de plus petites tailles, montrent à côté de silhouettes humaines rapidement esquissées, deux étonnantes figures, aux jambes fines et allongées et bras élancés, le ventre bombé, l'une avec un sexe masculin apparent, les visages simplifiés en têtes rondes. Maria Weyersberg, l'auteur de l'aquarelle, donna le nom de "Mantes religieuses " à ces êtres étranges aux membres d'insectes, qu'elle assimilait à des êtres divins. Recouvrant les parois de granit des monts de Spitzkoppe, elle les attribua aux Bochimans lors de son expédition en Namibie en 1928-1930. Quant à l'aquarelle sur papier d'Agnès Schulz, située dans la salle d'exposition à proximité, elle offre aussi au regard, en son centre, une figure humaine à la tête animale, le corps entouré de bandelettes, "lévitant » à l'horizontal, sexe éjaculant, tête, bras et jambes attirés vers le haut. Elle est entourée d'un gisant informe, en bas à droite, adoptant pratiquement la même pose que cette figure centrale, et en haut à gauche, d'un personnage aux jambes enveloppées, semblant s'envoler. Leo Frobenius dénomma ce site de Rusape, sur l'actuel territoire du Zimbabwe, « les monuments du roi » et en donna une interprétation symbolique, celle de l'investiture d'un nouveau roi. 
ill. 12 : Salle 1 de l'exposition, au centre, Maria Weyersberg, Combats de buffles d'Enfouss, 1912-1914, Huile sur toile, 63,7 x $141 \mathrm{~cm}$, Copie d'art rupestre faite à Enfouss, Djebel Amour, Algérie.

8 Le combat de buffles antiques d'Enfouss (ill.12) est la plus ancienne des toiles d'art rupestre présentée dans l'exposition, puisqu'elle fut établie lors de l'expédition de 1912-1914 en Algérie dans le Djebel Amour. Son auteur, Maria Weyersberg, avait sans doute réalisé cette huile sur toile également en plus grand format comme l'attestent des photographies du MoMA où elle occupait l'un des murs de l'exposition organisée à NewYork par Alfred H. Barr en 1937. Ces gravures rupestres de l'Atlas sont d'un grand réalisme, représentant par une simple fente dans le rocher, les corps de ces puissants animaux se combattant, cornes contre cornes, tandis qu'un homme, tourné vers la gauche, observe la scène et semble toucher l'animal. Avec une grande précision, ces bubalus antiquus sont figurés de profil, avec seulement les deux pattes visibles par le spectateur, leurs ergots à l'arrière des sabots, leur sexe, la queue fouettant la croupe. Leurs têtes avec la barbiche, les yeux et les cornes aux fines cannelures sont, quant à elles, représentées de face. Dans l'huile sur toile, comme sur les rochers, toutes les figures apparaissent superposées, le spectateur ne pouvant déterminer lequel des deux buffles est au premier plan et si l'homme était situé à l'avant ou à l'arrière de l'animal, le torse étant présenté de face et les bras de profil, la queue d'un autre buffle posée sur son épaule.

9 Si l'exposition présentait l'art rupestre africain à travers les images produites par l'Institut de Francfort dans la première moitié du XXe siècle, l'approche ne se voulait pas pour autant archéologique. Elle mettait plutôt en exergue le statut ambigu de ces images à travers leur histoire. 


\section{2. À la croisée de l'art et de la science, des images aux statuts ambigus}

10 Dès la première salle, l'histoire de ces images souligne en effet tant leurs valeurs scientifiques qu'artistiques. Traces de peintures et gravures d'âge préhistorique, ces images restituent des représentations, aujourd'hui parfois dégradées ou disparues, telles qu'elles existaient dans la première moitié $d u X X^{e}$ siècle. Contrairement à leurs homologues des grottes européennes, ces peintures à l'air libre ont subi l'érosion due aux conditions climatiques, aux fouilles mêmes et à une fréquentation touristique accrue, et plus récemment aux détériorations volontaires de mouvements intégristes religieux. Si certaines d'entre elles bénéficient d'un classement au patrimoine de l'humanité ou sont intégrées à des parcs naturels nationaux, des politiques efficaces de préservation et de restauration des sites sont aujourd'hui nécessaires. Les collections de l'Institut de Francfort sont donc d'autant plus précieuses, que ces relevés sont globalement conformes aux tailles et couleurs des images originales. En attestent les photographies prises par Leo Frobenius présentées également dans l'exposition, invitant le spectateur à une rapide comparaison avec les copies d'art rupestre. Les gravures rupestres furent d'abord soulignées à la craie avant d'être dessinées, photographiées ou peintes; les peintures rupestres furent, quant à elles, copiées à l'aide de calques et reportées sur des papiers, ensuite assemblés pour former des aquarelles de grands formats (ill. 8). La démarche était ici similaire à celles des archéologues effectuant des moulages des bas-reliefs des temples bouddhiques de l'Asie, substituts d'œuvres qui ne pouvaient être rapportées en Europe.

11 L'absence de signature sur ces toiles prouve d'ailleurs que les peintres, à l'origine des copies d'art rupestre, accordaient avant tout une valeur scientifique à cette documentation ethnographique. Certains étaient reconnus à l'époque, tel l'expressionniste abstrait Wolfgang Schulze (Wols), et trouvaient dans ces images préhistoriques des sources d'inspiration pour leur propre création artistique. La plupart était cependant des artistes méconnus, des femmes, formées dans des écoles de BeauxArts. Maria Weyersberg, Agnès S. Schultz, Elisabeth Pauli, Elisabeth Krebs, Elisabeth Mannsfeld ou Ruth Assisa Cuno accompagnèrent ainsi Leo Frobenius lors de ces expéditions dans l'intérieur de l'Afrique. Issues souvent de familles aisées, elles étaient aptes à s'offrir les frais de voyages, sans exiger un quelconque contrat pour participer à ces aventures. Dans l'Entre-deux-guerres, il était encore difficile pour une femme d'exercer la profession d'ethnologue ou d'archéologue, hors de l'anonymat, du bénévolat et sans être dans l'ombre de la gent masculine. Si elles étaient peu souvent mentionnées dans les publications, ces femmes furent pourtant à la source des productions visuelles et des expositions de l'Institut de morphologie culturelle fondé par Frobenius. Maria Weyersberg eut par exemple un rôle déterminant dans la préparation des expositions parisiennes et a entretenu une correspondance active avec Georges-Henri Rivière dans les années $1930 .^{3}$ Elles trouvèrent sans doute grâce à ces expéditions africaines un statut et une liberté sociale auxquels elles n'avaient pas accès en Allemagne, et c'est ainsi un regard essentiellement féminin qui fut posé sur ces images préhistoriques.

12 Toutefois, c'est bien en tant qu'œuvres d'art que ces images rupestres furent progressivement montrées, tels des originaux venant du passé. Les artistes de l'Institut de Francfort s'effacèrent alors volontiers devant ceux de la préhistoire. Plusieurs expositions furent organisées : en 1912, par la chambre des députés de Berlin, en 1913 par le Karl- 
Ernst Osthaus Museum de Hagen, en 1930 à la salle Pleyel de Paris, en 1931 à Vienne par le Hagenbund, en 1932 par l'Institut de morphologie culturelle de Francfort, en 1933 par le Musée d'ethnographie du Trocadéro de Paris, en 1936 au Bundespalais de Berlin, en 1937 au MoMA de New-York, ainsi que dans vingt-huit villes américaines. Leurs scénographies montrent l'ambiguïté du statut de ces images, l'art préhistorique n'étant alors connu que par ses reproductions, et le plus souvent en noir et blanc. Les copies d'art rupestre furent tantôt présentées comme des archives parallèlement aux collections de silex rapportées d'Afrique du Sud par l'abbé Breuil à la salle Pleyel en 1930 (ill.13), ou à l'appui d'une solide documentation scientifique au musée d'ethnographie du Trocadéro en 1933, parallèlement à l'ouverture de la salle de „préhistoire exotique“. Au contraire, Alfred H. Barr les présenta au MoMA à travers une mise en scène innovante dans des cubes blancs mettant en valeur la monumentalité et les couleurs de ces œuvres d'art. La brochure de l'exposition affichait clairement l'authenticité de ces images, assimilant ces imitations à des originaux et les comparant aux productions artistiques contemporaines (ill. 14).

ill. 13 : L'Afrique, Cahiers d'art, 1930

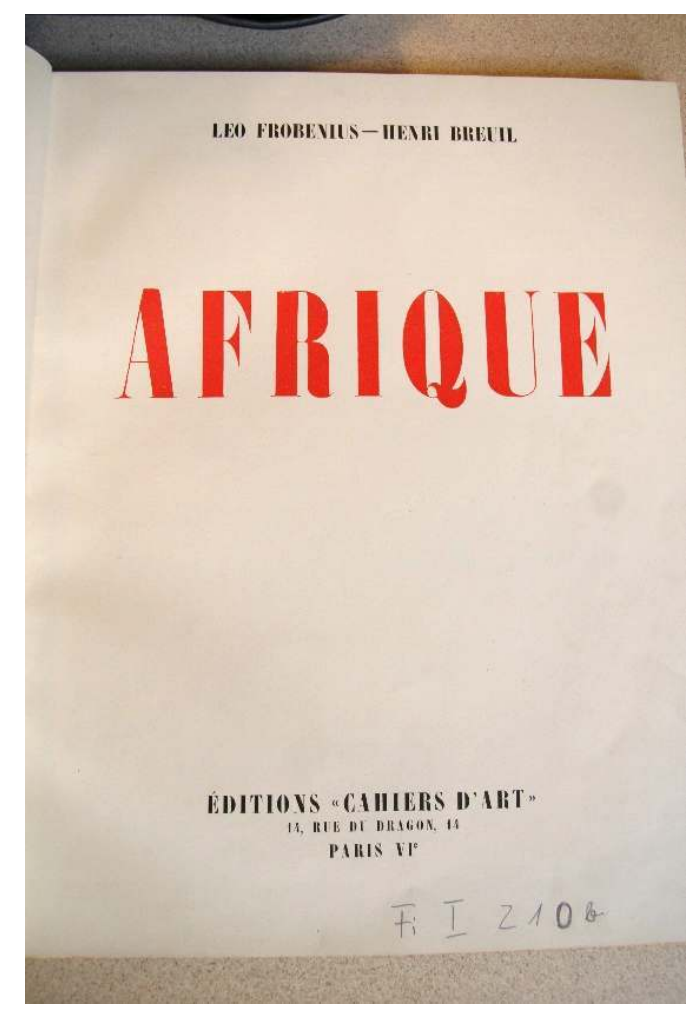




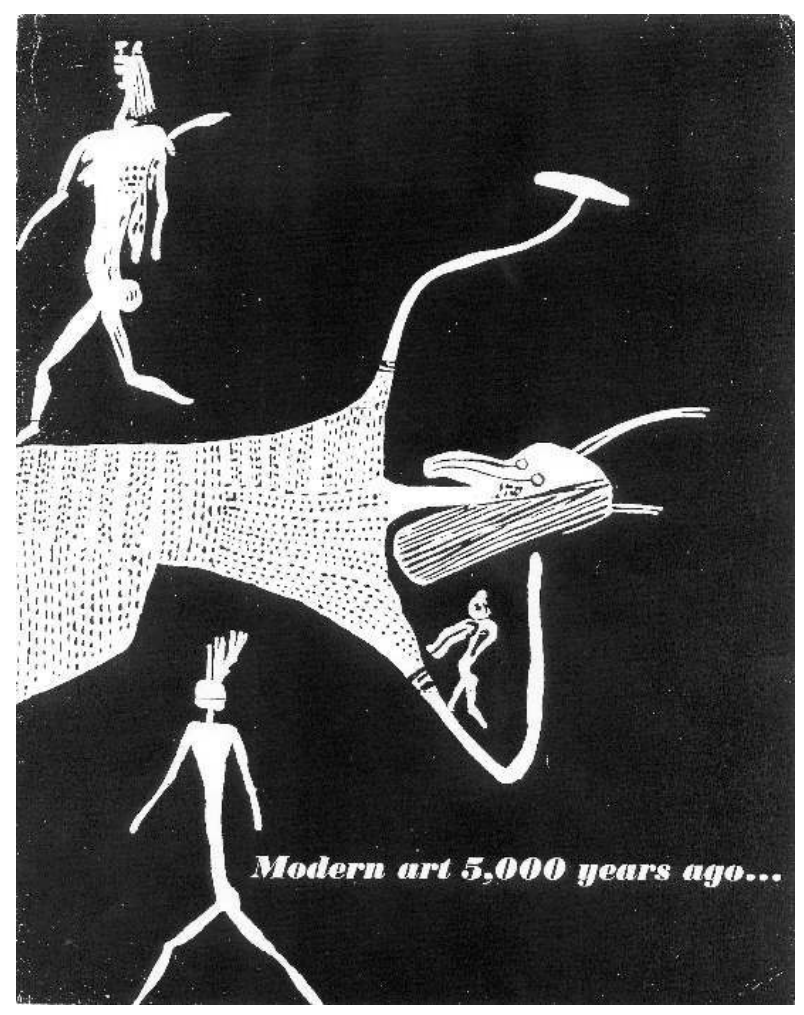

L'exposition de l'Institut Goethe soulignait à ce propos l'étroite relation établie dans l'Entre-deux-guerres entre art moderne et art préhistorique et la réception des expositions de l'Institut de Francfort par les artistes de l'avant-garde et les critiques littéraires. Elle présentait par exemple dans des vitrines les cartons de vernissage adressés à l'ensemble de l'avant-garde parisienne lors de l'exposition de 1930. La liste des invités dressée par le musée comprenait des noms aussi célèbres que ceux de Miró, Derain, Picasso, Marcoussis, Stravinsky, mais aussi Taittinger, Citroën ou Maspero. Elle citait aussi les critiques d'art qui s'étonnèrent des analogies formelles existant entre art préhistorique et art moderne. Certains artistes recherchèrent effectivement de nouvelles formes et conceptions artistiques dans ces images rupestres. Ernst Ludwig Kirchner, fondateur en 1905 du mouvement die Brücke, à l'origine de l'expressionnisme allemand, possédait trois des principales publications de Leo Frobenius dans sa bibliothèque. Willi Baumeister, peintre représentatif de l'expressionnisme abstrait, intégra plusieurs motifs provenant des collections de l'Institut de Francfort dans ses œuvres ${ }^{4}$. En 1937, Alfred H. Barr le constate : «L'art du XXe siècle est advenu sous l'influence de la grande tradition de l'art mural préhistorique ». À cet égard, le documentaire d'Arte Metropolis, présenté dans la première salle du Goethe-Institut, La peinture rupestre et l'art moderne américain, apportait un éclairage sur les recherches en cours concernant l'exposition du MoMA et sa réception ${ }^{5}$. Plusieurs artistes majeurs, entre autres Jackson Pollock et Willem de Kooning, virent probablement l'exposition de New York, et des œuvres de Paul Klee, André Masson, Joan Miró, Jean Arp, Wassily Kandinsky, Vladimir Lebedev ou Mikhail Larionow furent exposées simultanément aux relevés d'art rupestre ${ }^{6}$. Après avoir organisé les expositions African Negro Art et Cubism and Abstract art, l'exposition Prehistoric rock pictures from Europe and Africa venait ainsi consacrer les liens entre primitivisme et modernité. 
Simples fac-similés des peintures et gravures rupestres, les copies de l'Institut Frobenius ont donc acquis le statut d'œuvres d'art originales, par la scénographie des expositions de l'Entre-deux-guerres et leur assimilation à l'art moderne contemporain, en hommage d'un art préhistorique qui, lui, ne pouvait être exposé.

\section{Polémiques autour des collections de Leo Frobenius : pratiques et théories en question.}

La seconde partie de l'exposition était consacrée aux pratiques ethnographiques et théories anthropologiques développées par Leo Frobenius. En rupture avec les conceptions évolutionnistes de son temps, Leo Frobenius contribua au contraire au développement de théories diffusionnistes. Mises en avant par Friedrich Ratzel, Bernhard Ankermann et Fritz Graebner, ses théories prônaient l'idée d'une migration des formes culturelles à partir de différents foyers de civilisation. Ses expéditions africaines lui apportèrent ensuite la conviction que l'Afrique portait les traces de l'ancienne Atlantide. Découvrant lors de son expédition de 1910-1912 dans la colonie britannique du Nigéria des sculptures antiques en bronze et en terres cuites d'une étonnante beauté, il affirma qu'elles étaient la preuve de la véracité de ce mythe et gagna ainsi une popularité auprès du grand public. Il poursuivit ensuite cette quête des formes originelles de la culture à travers la copie des gravures et peintures rupestres des temps préhistoriques. Pragmatique, l'explorateur fut toujours proche du pouvoir et obtint l'appui financier et politique de partis hétéroclites. Son amitié avec Guillaume II lui permit d'organiser ses premières expéditions. Sa pensée conservatrice explique qu'il ait maintenu des liens avec l'empereur déchu lors de son exil à la Haus Doorn aux Pays-Bas. Leo Frobenius repartit ensuite dans les années 1930 dans le Sahara avec le soutien du régime nazi, mais aussi celui du régime fasciste de Mussolini. Bien que ses théories anthropologiques, par le primat qu'elles accordent à la notion de culture sur celle de communauté d'individus et de races, soient fondamentalement opposées à la pensée nazie, ses premières publications sont empreintes de racisme, comme beaucoup d'autres au début du siècle. L'Institut de morphologie culturelle n'est pas non plus dénué de compromissions avec les régimes fascistes en place, sans l'accord desquels l'africaniste n'aurait pu organiser ses expéditions, notamment dans la colonie italienne en Libye ou ses expositions d'art rupestre, telle celle de New-York en 1937. Toutefois, les nazis auraient voulu fermer l'Institut à la mort de Frobenius en 1938.

La réception enthousiaste de ses œuvres par les intellectuels africains des années 1930 prouve effectivement que c'est une image de l'Afrique bien différente de celle donnée par les théories raciales et coloniales de l'époque que Leo Frobenius diffusait sur le vieux continent. Les escaliers menant à la dernière salle de l'exposition s'en font l'écho (ill. 6). Frobenius suscita l'admiration d'Aimé Césaire et de Léopold Sédar Senghor pour sa valorisation d'une civilisation et d'une âme africaine, idées qui contribuèrent à poser les fondements de la pensée du mouvement de la négritude. L'abbé Breuil allait même jusqu'à le qualifier d'«apôtre» propageant «foi dans la science» et créant une «bibliothèque de pierre » de la préhistoire africaine. Son Histoire de la civilisation africaine lui valut aussi les éloges des activistes des droits civiques aux États-Unis, à l'instar de l'historien W. E. B. Dubois. Sa transcription des contes issus des traditions orales attira, quant à elle, l'intérêt des écrivains, tandis que ses relevés d'art rupestre suscitèrent la curiosité des artistes et critiques contemporains. Rejetés par les institutions académiques 
pour l'impétuosité de son caractère et sa philosophie «fantasque » de la culture, Leo Frobenius parvint ainsi par son active politique d'expositions et de vulgarisation du savoir à sensibiliser une élite intellectuelle et artistique à ses recherches. Les critiques se firent par la suite plus vives. À l'époque postcoloniale, les ethnologues et archéologues furent regardés comme des colons européens, responsables du pillage de l'Afrique. L'écrivain malien, Yambo Ouologuem, dressa dans son roman Le devoir de violence une sévère satire d'un ethnologue allemand débarqué en terre africaine, Fritz Schrobenius. Sous ces traits, on reconnait le portrait à charge de Leo Frobenius, avide collectionneur, rusé et cupide, accusé de spolier le patrimoine africain. Au-delà, il critiqua la pensée conservatrice de l'ethnologue qui voulait «ressusciter, sous couleur d'autonomie culturelle, un univers africain qui ne correspondait à plus rien de vivant». Quant à l'écrivain nigérian, Wole Soyinka, il releva les propos racistes de The Voice of Africa et montra le mépris avec lequel Leo Frobenius traitait les populations « évoluées » de cette colonie britannique, même s'il admirait leur culture passée.

La seconde salle présentait l'étendue et la variété des collections actuelles de l'Institut Frobenius de Francfort. L'exposition y décrivait le cadre colonial spécifique dans lequel les expéditions ethnographiques furent organisées. Elle rappelait aussi comment elles eurent pour conséquence l'éveil d'une conscience patrimoniale, tant de la part des administrations coloniales que des populations locales. Les collectes massives d'artefacts du début du siècle, puis de la littérature orale africaine, laissèrent place dans l'Entredeux-guerres à l'établissement, moins polémique, de relevés d'art rupestre. Concernant cette période, l'Institut Frobenius n'est par conséquent aujourd'hui en possession que de copies et de moulages. Pour financer ses recherches et expéditions, Leo Frobenius a vendu de son vivant les collections ethnographiques rassemblées en Afrique, notamment aux musées d'ethnographie allemands. Ces derniers doivent aujourd'hui faire face aux revendications croissantes des populations autochtones vis-à-vis de collections devenues sensibles. Conservés à l'Institut Frobenius de Francfort, les films ethnographiques, les comptes-rendus d'expédition, les notes et fiches, dessins, peintures et photographies sont, quant à eux, des sources pour l'histoire coloniale, l'histoire des explorations et l'histoire des sciences. L'authenticité de certaines séquences filmées peut certes être mise en doute, car elles donnaient lieu à l'époque à de véritables reconstitutions et mises en scène. Les films projetés ${ }^{7}$ lors de l'exposition montrent toutefois la vie quotidienne des populations locales du royaume lozi, la hiérarchie sociale existant dans cette cour et les danses réunissant le village à la fin des années 1920. Ils dévoilent aussi les conditions précaires de ces expéditions dans le Sahara avec les premières jeeps de Ford au début des années 1930, embarquant le matériel nécessaire aux semaines d'isolement dans le désert. 
Enfin, l'exposition rendait aussi compte du travail quotidien des artistes lors des expéditions. Escaladant les falaises, debout sur des échelles le long des parois ou sous les abris rocheux, assis devant le chevalet, ils observaient avec minutie les détails des peintures et gravures pour les reporter sur le papier. L'une des vitrines présentait d'ailleurs le matériel laissé derrière eux, dans les années 1930, récemment retrouvé par des archéologues dans le Sahara libyen ${ }^{8}$. Dans les autres vitrines étaient rassemblées les nombreuses publications de Leo Frobenius réunissant les relevés d'art rupestre et ses atlas de morphologie culturelle (ill.15). Elles ne sont pas sans rappeler d'autres entreprises contemporaines de collectes d'images, tel l'Atlas mnémosyne d'Aby Warburg. Des documents d'archives, relatifs aux expositions de l'Institut, attestaient de leur réception auprès de l'avant-garde parisienne et du grand public. Cette dernière salle exposait enfin des photographies et dessins à l'encre de Tombouctou de Fritz Nansen en 1909 (ill. 16). Ils représentaient l'architecture typique de villes musulmanes et les maisons des premiers explorateurs européens, tels René Caillé ou Heinrich Barth. Ils inscrivaient ainsi les expéditions de Leo Frobenius dans la continuité des explorations scientifiques du XIXe siècle. Comme face aux relevés d'art rupestre, et à certaines photographies, le spectateur ne saurait toutefois se limiter à cet aspect documentaire. Ce serait trop vite oublier le talent des photographes et peintres travaillant pour l'Institut de Francfort dans l'Entre-deux-guerres. S'ils recherchaient la fidélité aux modèles des temps passés, ils n'en furent pas moins capables de réaliser des copies sur papier qui laissaient transparaître la beauté des originaux de pierre et parfois même les surpassèrent. 


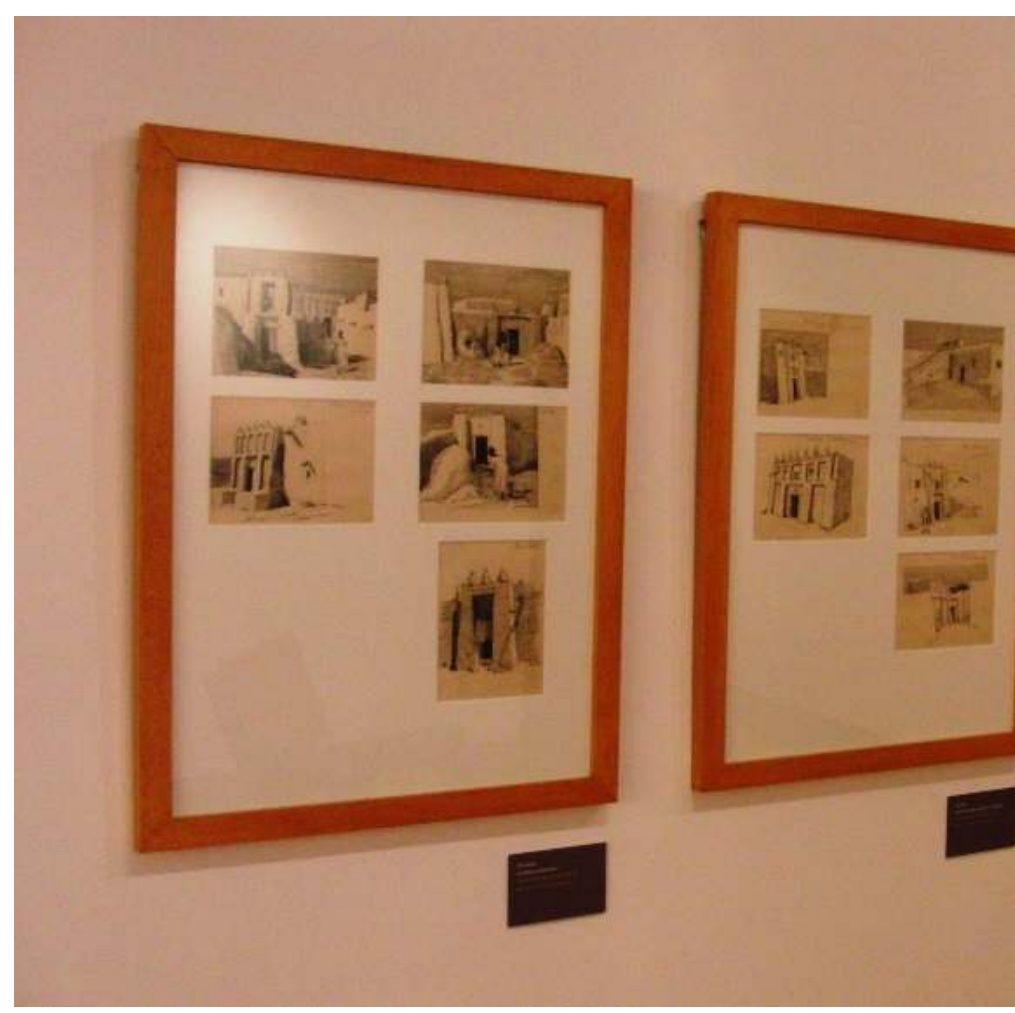

Le classement de la grotte Chauvet au patrimoine de l'humanité est récemment venu rappeler la nécessité de préserver ce fonds commun d'images du passé (ill. 17). Certes, les relevés d'art rupestre ne sont que des copies. Et cependant, ce sont bien souvent les seules traces conservées de gravures et peintures originales parfois dégradées ou menacées. Dès les années 1930, Leo Frobenius avait saisi cet enjeu politique et scientifique. À travers ses expositions, il témoignait dans une Europe coloniale de l'existence d'une civilisation et d'une préhistoire commune à l'humanité. Ce n'était sans doute ici que le prolongement de ses théories sur l'Atlantide. Bien sûr, les expositions de l'Institut de Francfort eurent lieu parallèlement à l'inauguration de la salle de préhistoire exotique, comme si pour George-Henri Rivière ou Paul Rivet, il existait une préhistoire des Autres, différente de celle de l'Europe. Toutefois, on ne saurait trop souligner la rupture que constituait déjà l'invention d'une préhistoire africaine, dans une époque coloniale, refusant à ces peuples le droit même d'avoir une histoire et une culture aussi dignes d'intérêt que celles de l'Europe. La valeur artistique des relevés n'échappa pas non plus à l'avant-garde européenne, et même américaine, grâce à des expositions comme celles du MoMA dont Alfred H. Barr et Douglas Fox furent les promoteurs, plus que Leo Frobenius. Le spectateur d'aujourd'hui ne peut encore que s'émerveiller devant la beauté de certaines toiles, dessins à l'encre et photographies dont la monumentalité, le graphisme et les dégradés de couleurs ne cessent de provoquer la surprise. 


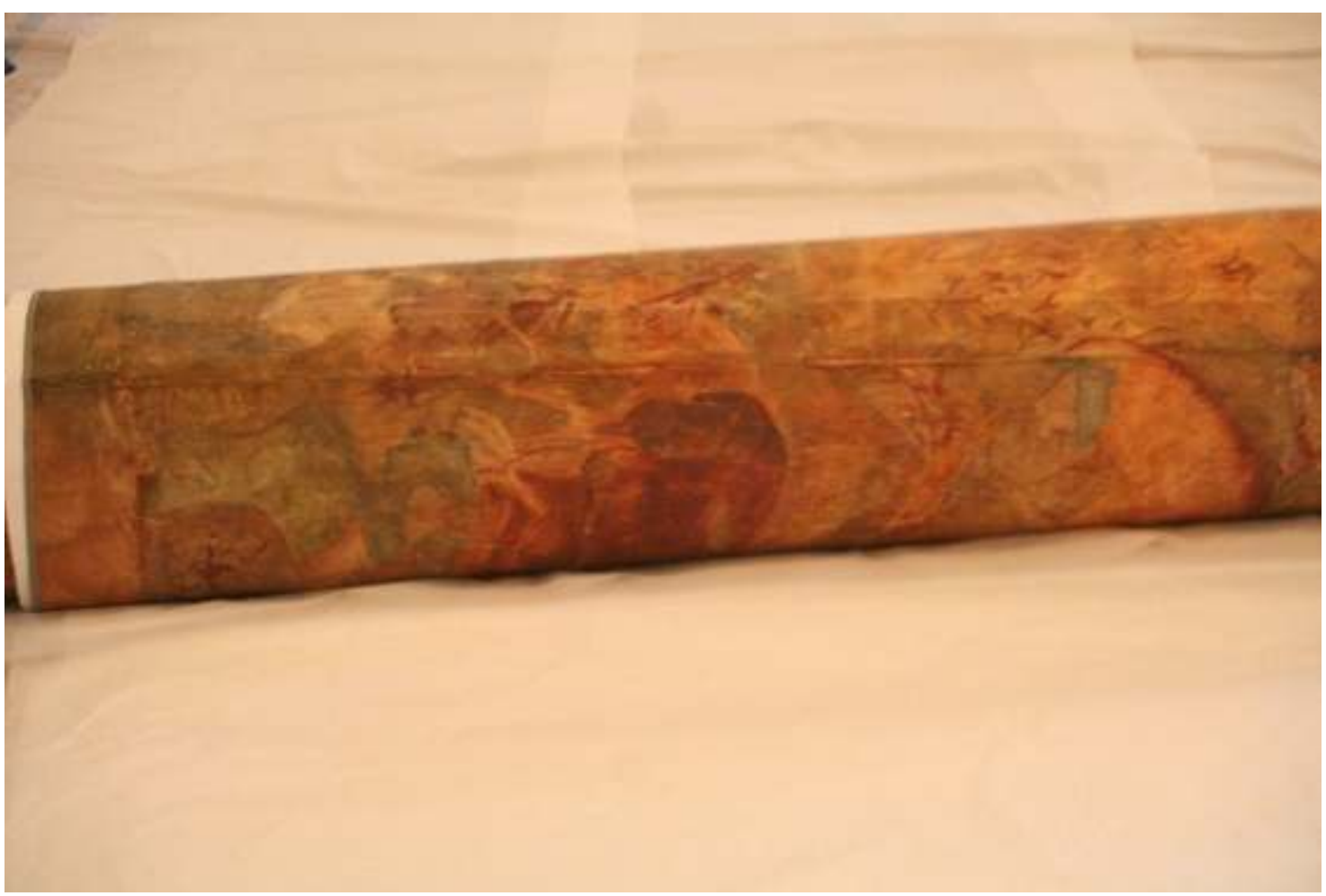

\section{NOTES}

1. Les résumés des interventions sont accessibles sur le site suivant: http:// frobenius2014.sciencesconf.org/

2. Sur les expositions parisiennes de Leo Frobenius à la salle Pleyel en 1930 et au musée d'ethnographie du Trocadéro en 1933, voir Hélène Ivanoff, « Exposer l'art rupestre à Paris dans les années 1930 », Studien zur Kulturkunde, à paraître en 2015.

3. Hélène Ivanoff, « Exposer l'art rupestre à Paris dans les années 1930 », Studien zur Kulturkunde, à paraître en 2015.

4. Viola Hildebrandt-Schat, " Die Frankfurter Jahre 1928 - 1933 », Ausst. Kat. Willi Baumeister (1889-1955). Die Frankfurter Jahre 1928-1933. Zum 50. Todestag, Frankfurt am Main, 2005, p.11-17.

5. «Afrikanische Höhlenmalerei und die moderne Kunst in Amerika », «La peinture rupestre et l'art moderne américain », Réalisatrice : Christine Romann, Productrice : Hessischer Rundfunk, Arte Metropolis.

6. Elke Seibert, «Prähistorische Malerei im Museum of Modern Art in New-York 1937 », Kunst Text.de 2/2014-1 (http://edoc.hu-berlin.de/kunsttexte/2014-2/seibert-elke-3/PDF/seibert.pdf).

7. Les deux extraits de films présentés sont des séquences concernant l'expédition de 1928-1930 en Afrique du Sud et de 1932 dans le désert libyen.

8. Sur cette découverte archéologique, voir: http://www.heritagedaily.com/2014/06/thewonderful-rubbish-of-the-gilf-kebir-desert/103662. 


\section{AUTEUR}

\section{HÉLÈNE IVANOFF}

(UMR 8131, CNRS/EHESS)

Hélène Ivanoff a été lauréate de la bourse Gabriel Monod de l'IFHA en 2013. 